\title{
Analogues of Koshliakov's formula
}

\author{
Bruce C. Berndt, Sun Kim, and Alexandru Zaharescu \\ In Memory of Srinivasa Ramanujan
}

Abstract. Two character analogues of the ordinary divisor function $d(n)$ are defined. For each, a character analogue of Koshliakov's formula is proved. An application is made to the positivity of $L(1, \chi)$ for even real characters $\chi$.

\section{Introduction}

Deserving of more respect, the Russian mathematician, N. S. Koshliakov, is chiefly remembered for one theorem that bears his name, Koshliakov's formula $\mathbf{9}$. To state his formula, let $K_{0}(z)$ denote the modified Bessel function of order 0 , which can be defined by [12, p. 80]

$$
K_{0}(z)=-\log \left(\frac{1}{2} z\right) \cdot I_{0}(z)+\sum_{m=0}^{\infty} \frac{\left(\frac{1}{2} z\right)^{2 m}}{(m !)^{2}} \psi(m+1),
$$

where

$$
I_{0}(z)=\sum_{m=0}^{\infty} \frac{\left(\frac{1}{2} z\right)^{2 m}}{(m !)^{2}},
$$

and where $\psi(z)$ denotes the logarithmic derivative of the gamma function, i.e.,

$$
\psi(z)=\frac{\Gamma^{\prime}(z)}{\Gamma(z)} .
$$

Next, let $d(n)$ denote the number of positive divisors of the positive integer $n$. If $\gamma$ denotes Euler's constant, Koshliakov's formula is given by

$$
\begin{aligned}
& \sqrt{\alpha}\left(\frac{1}{4} \gamma-\frac{1}{4} \log (4 \beta)+\sum_{n=1}^{\infty} d(n) K_{0}(2 n \alpha)\right) \\
& \quad=\sqrt{\beta}\left(\frac{1}{4} \gamma-\frac{1}{4} \log (4 \alpha)+\sum_{n=1}^{\infty} d(n) K_{0}(2 n \beta)\right),
\end{aligned}
$$

where $\alpha$ and $\beta$ denote positive numbers such that $\alpha \beta=\pi^{2}$. Koshliakov's proof, as well as most subsequent proofs, depends upon Voronoï's summation formula.

2010 Mathematics Subject Classification. Primary: 33C10; Secondary: 11M06.

Key words and phrases. Koshliakov's formula, Dirichlet characters, Dirichlet $L$-functions, Dirichlet's theorem on primes in arithmetic progressions, modified Bessel functions.

The first author's research was partially supported by NSA grant H98230-11-1-0200. 
Koshliakov's formula can be regarded as an analogue of the transformation formula for the classical theta function, namely,

$$
\sum_{n=-\infty}^{\infty} e^{-\pi n^{2} / \tau}=\sqrt{\tau} \sum_{n=-\infty}^{\infty} e^{-\pi n^{2} \tau}, \quad \operatorname{Re} \tau>0,
$$

which, as is well known, is equivalent to the functional equation of the Riemann zeta function $\zeta(s)$ given by [11, p. 22]

$$
\pi^{-s / 2} \Gamma\left(\frac{1}{2} s\right) \zeta(s)=\pi^{-(1-s) / 2} \Gamma\left(\frac{1}{2}(1-s)\right) \zeta(1-s) .
$$

Koshliakov's formula (1.3) is equivalent to the functional equation of $\zeta^{2}(s)$.

In fact, Koshliakov's formula is not originally due to Koshliakov; it can be found in Ramanujan's lost notebook [10, p. 253], and so Ramanujan probably discovered it approximately ten years before Koshliakov rediscovered it. In contrast to Koshliakov's proof via the Voronoïsummation formula, Ramanujan derived Koshliakov's formula from Guinand's formula. For more information about Koshliakov's formula and for more details on its proofs, especially that of Ramanujan, see the paper by the first author, Y. Lee, and J. Sohn [3] or the first author's book with G. E. Andrews [1, Chapter 3].

The first purpose of this short note is to derive two character analogues of Koshliakov's formula (1.3). We note that S. Yakubovich 13 has recently established new generalizations of Koshliakov's formula, and the first author, A. Dixit, and J. Sohn 2 have derived character analogues of (1.3) in which the character $\chi(n)$ appears in the series coefficients multiplying $d(n)$. Our character analogues in this paper are different, although our proofs are in the spirit of those of W. L. Ferrar [8] and Yakubovich [13. In this paper, the divisor function $d(n)$ is replaced by a sum of characters summed over divisors of $n$. More precisely, if $\chi$ denotes any character modulo $q$, we define

$$
d_{\chi}(n):=\sum_{d \mid n} \chi(d)
$$

Moreover, if $\chi_{1}$ and $\chi_{2}$ denote arbitrary characters modulo $p$ and $q$, respectively, we define

$$
d_{\chi_{1}, \chi_{2}}(n):=\sum_{d \mid n} \chi_{1}(d) \chi_{2}(n / d)
$$

It is easy to see that the generating functions for these two arithmetical functions are, respectively,

$$
\zeta(2 s) L(2 s, \chi)=\sum_{n=1}^{\infty} d_{\chi}(n) n^{-2 s}, \quad \sigma=\operatorname{Re} s>\frac{1}{2},
$$

and

$$
L\left(2 s, \chi_{1}\right) L\left(2 s, \chi_{2}\right)=\sum_{n=1}^{\infty} d_{\chi_{1}, \chi_{2}}(n) n^{-2 s}, \quad \sigma>\frac{1}{2},
$$

where $L(s, \chi)$ is the Dirichlet $L$-function associated with the character $\chi$. 
The second purpose of our paper is to show that one of our analogues of Koshliakov's formula (Theorem 3.1) implies the positivity of $L(1, \chi)$ for even real characters, a crucial fact in proving Dirichlet's theorem on primes in arithmetic progressions.

\section{Preliminary Results}

We recall that if $\chi$ is a nonprincipal even primitive character of modulus $q$, and if $\tau(\chi):=\sum_{n(\bmod q)} \chi(n) e^{2 \pi i n / q}$ denotes the Gauss sum, then $L(x, \chi)$ satisfies the functional equation [5, p. 69]

$$
\left(\frac{\pi}{q}\right)^{-s} \Gamma(s) L(2 s, \chi)=\frac{\tau(\chi)}{\sqrt{q}}\left(\frac{\pi}{q}\right)^{-\left(\frac{1}{2}-s\right)} \Gamma\left(\frac{1}{2}-s\right) L(1-2 s, \bar{\chi}) .
$$

So, by (1.4) and (2.1),

$$
\begin{aligned}
& \left(\frac{\pi}{\sqrt{q}}\right)^{-2 s} \Gamma^{2}(s) \zeta(2 s) L(2 s, \chi) \\
& \quad=\frac{\tau(\chi)}{\sqrt{q}}\left(\frac{\pi}{\sqrt{q}}\right)^{-2\left(\frac{1}{2}-s\right)} \Gamma^{2}\left(\frac{1}{2}-s\right) \zeta(1-2 s) L(1-2 s, \bar{\chi}),
\end{aligned}
$$

and, for even nonprincipal primitive characters $\chi_{1}$ and $\chi_{2}$ modulo $p$ and $q$, respectively,

$$
\begin{aligned}
& \left(\frac{\pi^{2}}{p q}\right)^{-s} \Gamma^{2}(s) L\left(2 s, \chi_{1}\right) L\left(2 s, \chi_{2}\right) \\
& \quad=\frac{\tau\left(\chi_{1}\right) \tau\left(\chi_{2}\right)}{\sqrt{p q}}\left(\frac{\pi^{2}}{p q}\right)^{-\left(\frac{1}{2}-s\right)} \Gamma^{2}\left(\frac{1}{2}-s\right) L\left(1-2 s, \overline{\chi_{1}}\right) L\left(1-2 s, \overline{\chi_{2}}\right) .
\end{aligned}
$$

Recall that if $\chi$ is an odd primitive character of modulus $q$, then $L(x, \chi)$ satisfies the functional equation [5, p. 71]

$$
\left(\frac{\pi}{q}\right)^{-\left(s+\frac{1}{2}\right)} \Gamma\left(s+\frac{1}{2}\right) L(2 s, \chi)=-\frac{i \tau(\chi)}{\sqrt{q}}\left(\frac{\pi}{q}\right)^{-(1-s)} \Gamma(1-s) L(1-2 s, \bar{\chi}) .
$$

Using (2.4), we see that, for odd primitive characters $\chi_{1}$ and $\chi_{2}$ modulo $p$ and $q$, respectively,

$$
\begin{aligned}
& \left(\frac{\pi^{2}}{p q}\right)^{-\left(s+\frac{1}{2}\right)} \Gamma^{2}\left(s+\frac{1}{2}\right) L\left(2 s, \chi_{1}\right) L\left(2 s, \chi_{2}\right) \\
& =-\frac{\tau\left(\chi_{1}\right) \tau\left(\chi_{2}\right)}{\sqrt{p q}}\left(\frac{\pi^{2}}{p q}\right)^{-(1-s)} \Gamma^{2}(1-s) L\left(1-2 s, \overline{\chi_{1}}\right) L\left(1-2 s, \overline{\chi_{2}}\right) .
\end{aligned}
$$

We shall also use the Laurent expansions [4, p. 215], [11, p. 16, equation

$$
\begin{aligned}
\Gamma(s) & =\frac{1}{s}-\gamma+O(s) \\
\zeta(s) & =\frac{1}{s-1}+\gamma+O(s-1)
\end{aligned}
$$

where $\gamma$ is Euler's constant. 
Recall that $K_{0}(x)$ is defined by (1.1). Crucial in our investigations is the inverse Mellin transform [7, p. 349, equation (17)]

$$
\frac{1}{2 \pi i} \int_{c-i \infty}^{c+i \infty} \Gamma^{2}(s) x^{-s} d s=2 K_{0}(2 \sqrt{x}), \quad c>0 .
$$

We also need the integral representation [12, p. 446]

$$
K_{0}(x)=\int_{0}^{\infty} e^{-x \cosh t} d t
$$

We note the asymptotic estimate [12, p. 202]

$$
K_{0}(x)=\left(\frac{\pi}{2 x}\right)^{1 / 2} e^{-x}+O\left(\frac{e^{-x}}{x^{3 / 2}}\right), \quad \text { as } x \rightarrow \infty .
$$

\section{Analogues of Koshliakov's Formula}

THEOREM 3.1. If $\chi$ denotes a nonprincipal even primitive character of modulus $q$, and if $\operatorname{Re} z>0$, then

$$
\frac{q L(1, \chi)}{4 \tau(\chi)}+\sum_{n=1}^{\infty} d_{\chi}(n) K_{0}\left(\frac{2 \pi n z}{\sqrt{q}}\right)=\frac{\sqrt{q} L(1, \chi)}{4 z}+\frac{\tau(\chi)}{z \sqrt{q}} \sum_{n=1}^{\infty} d_{\bar{\chi}}(n) K_{0}\left(\frac{2 \pi n}{z \sqrt{q}}\right) .
$$

Proof. By (2.8) and (1.7), for $c>\frac{1}{2}$,

$$
\begin{aligned}
I_{1}: & =\frac{1}{2 \pi i} \int_{c-i \infty}^{c+i \infty}\left(\frac{\pi}{\sqrt{q}}\right)^{-2 s} \Gamma^{2}(s) \zeta(2 s) L(2 s, \chi) z^{-2 s} d s \\
& =\sum_{n=1}^{\infty} d_{\chi}(n) \frac{1}{2 \pi i} \int_{c-i \infty}^{c+i \infty} \Gamma^{2}(s)\left(\frac{\pi^{2} n^{2} z^{2}}{q}\right)^{-s} d s \\
& =2 \sum_{n=1}^{\infty} d_{\chi}(n) K_{0}\left(\frac{2 \pi n z}{\sqrt{q}}\right) .
\end{aligned}
$$

We now move the line of integration $\sigma=c$ at the left-hand side of (3.2) to $\sigma=-b$, $0<b<1$, by integrating over a rectangle with vertices $c \pm i T$ and $-b \pm i T, T>0$, and letting $T \rightarrow \infty$. By Stirling's formula along a vertical line [4, p. 224]

$$
|\Gamma(\sigma+i t)| \sim \sqrt{2 \pi}|t|^{\sigma-\frac{1}{2}} e^{-\frac{1}{2} \pi|t|}, \quad|t| \rightarrow \infty,
$$

and the bounds [11, p. 81]

$$
\zeta(\sigma+i t)=O\left(|t|^{k_{1}}\right), \quad L(\sigma+i t)=O\left(|t|^{k_{2}}\right), \quad \sigma>\sigma_{0}
$$

for any fixed $\sigma_{0}>0$, the integrals over the horizontal sides of this rectangle tend to 0 as $T$ tends to infinity. In applying the residue theorem, we note that the integrand has poles at $s=0$ and $s=\frac{1}{2}$. Let $R_{a}$ denote the residue at a pole $a$. Since $\chi$ is even, $L(0, \chi)=0$; also $\Gamma(s)$ has a simple pole at $s=0$ (see (2.6) $)$. Since $\zeta(0)=-\frac{1}{2}$ [11. p. 19], we find that

$$
R_{0}=-\frac{1}{2} L^{\prime}(0, \chi)=-\frac{q}{2 \tau(\chi)} L(1, \chi)
$$


where we have used an equality of C. Deninger [6, p. 182, equation (3.5)]. To calculate the residue at $s=\frac{1}{2}$ arising from the simple pole of $\zeta(2 s)$ at $s=\frac{1}{2}$, we use (2.7) and recall that $\Gamma\left(\frac{1}{2}\right)=\sqrt{\pi}$. Thus,

$$
R_{1 / 2}=\left(\frac{\pi z}{\sqrt{q}}\right)^{-1} \pi \cdot \frac{1}{2} L(1, \chi)=\frac{\sqrt{q}}{2 z} L(1, \chi) .
$$

Summarizing our analysis above and using (3.2)-(3.4), we find that

$$
\begin{aligned}
I_{1}= & 2 \sum_{n=1}^{\infty} d_{\chi}(n) K_{0}\left(\frac{2 \pi n z}{\sqrt{q}}\right) \\
= & -\frac{q}{2 \tau(\chi)} L(1, \chi)+\frac{\sqrt{q}}{2 z} L(1, \chi) \\
& +\frac{1}{2 \pi i} \int_{-b-i \infty}^{-b+i \infty}\left(\frac{\pi}{\sqrt{q}}\right)^{-2 s} \Gamma^{2}(s) \zeta(2 s) L(2 s, \chi) z^{-2 s} d s \\
= & : \frac{\sqrt{q}}{2}\left(\frac{1}{z}-\frac{\sqrt{q}}{\tau(\chi)}\right) L(1, \chi)+I_{2} .
\end{aligned}
$$

We now must analyze $I_{2}$.

Using the functional equation (2.2), making the change of variable $w=\frac{1}{2}-s$, inverting the order of summation and integration by absolute convergence (since $b>0$ ), and employing the inverse Mellin transform (2.8), we find that

$$
\begin{aligned}
I_{2} & =\frac{1}{2 \pi i} \int_{-b-i \infty}^{-b+i \infty} \frac{\tau(\chi)}{\sqrt{q}}\left(\frac{\pi}{\sqrt{q}}\right)^{-2\left(\frac{1}{2}-s\right)} \Gamma^{2}\left(\frac{1}{2}-s\right) \zeta(1-2 s) L(1-2 s, \bar{\chi}) z^{-2 s} d s \\
& =\frac{\tau(\chi)}{z \sqrt{q}} \frac{1}{2 \pi i} \int_{\frac{1}{2}+b-i \infty}^{\frac{1}{2}+b+i \infty}\left(\frac{\pi}{\sqrt{q}}\right)^{-2 w} \Gamma^{2}(w) \zeta(2 w) L(2 w, \bar{\chi}) z^{2 w} d w \\
& =\frac{\tau(\chi)}{z \sqrt{q}} \sum_{n=1}^{\infty} d_{\bar{\chi}}(n) \frac{1}{2 \pi i} \int_{\frac{1}{2}+b-i \infty}^{\frac{1}{2}+b+i \infty} \Gamma^{2}(w)\left(\frac{\pi n}{z \sqrt{q}}\right)^{-2 w} d w
\end{aligned}
$$

$$
=\frac{2 \tau(\chi)}{z \sqrt{q}} \sum_{n=1}^{\infty} d_{\bar{\chi}}(n) K_{0}\left(\frac{2 \pi n}{z \sqrt{q}}\right) .
$$

If we employ (3.6) in (3.5), we complete the proof of Theorem 3.1 .

THEOREM 3.2. Let $\chi_{1}$ and $\chi_{2}$ denote nonprincipal even primitive characters modulo $p$ and $q$, respectively. Then

$$
\sum_{n=1}^{\infty} d_{\chi_{1}, \chi_{2}}(n) K_{0}\left(\frac{2 \pi n z}{\sqrt{p q}}\right)=\frac{\tau\left(\chi_{1}\right) \tau\left(\chi_{2}\right)}{z \sqrt{p q}} \sum_{n=1}^{\infty} d_{\overline{\chi_{1}}, \overline{\chi_{2}}}(n) K_{0}\left(\frac{2 \pi n}{z \sqrt{p q}}\right) .
$$

PROOF. For $c>\frac{1}{2}$, upon inverting the order of summation and integration and invoking (2.8), we find that

$$
\begin{aligned}
I: & =\frac{1}{2 \pi i} \int_{c-i \infty}^{c+i \infty}\left(\frac{\pi^{2}}{p q}\right)^{-s} \Gamma^{2}(s) L\left(2 s, \chi_{1}\right) L\left(2 s, \chi_{2}\right) z^{-2 s} d s \\
& =\sum_{n=1}^{\infty} d_{\chi_{1}, \chi_{2}}(n) \frac{1}{2 \pi i} \int_{c-i \infty}^{c+i \infty} \Gamma^{2}(s)\left(\frac{\pi^{2} n^{2} z^{2}}{p q}\right)^{-s} d s
\end{aligned}
$$




$$
=2 \sum_{n=1}^{\infty} d_{\chi_{1}, \chi_{2}}(n) K_{0}\left(\frac{2 \pi n z}{\sqrt{p q}}\right) .
$$

We now proceed as before and move the line of integration $\sigma=c$ at the left-hand side of (3.8) to $\sigma=-b, 0<b<1$, by integrating over a rectangle with vertices $c \pm i T$ and $-b \pm i T, T>0$, and letting $T \rightarrow \infty$. The argument is the same as that in the proof of Theorem [3.1, but now it is easier, since the integrand is analytic on the interior of the rectangle because $L\left(0, \chi_{1}\right)=0=L\left(0, \chi_{2}\right)$ and $\chi_{1}$ and $\chi_{2}$ are nonprincipal. Thus, by an argument analogous to that previously given, we find that

$$
I=2 \frac{\tau\left(\chi_{1}\right) \tau\left(\chi_{2}\right)}{z \sqrt{p q}} \sum_{n=1}^{\infty} d_{\overline{\chi_{1}}, \overline{\chi_{2}}}(n) K_{0}\left(\frac{2 \pi n}{z \sqrt{p q}}\right) .
$$

Combining (3.9) with (3.8), we complete the proof of Theorem 3.2 .

THEOREM 3.3. If $\chi_{1}$ and $\chi_{2}$ denote odd primitive characters with moduli $p$ and $q$, respectively, and if $\operatorname{Re} z>0$, then

$$
\sum_{n=1}^{\infty}(n z) d_{\chi_{1}, \chi_{2}}(n) K_{0}\left(\frac{2 \pi n z}{\sqrt{p q}}\right)=-\frac{\tau\left(\chi_{1}\right) \tau\left(\chi_{2}\right)}{z \sqrt{p q}} \sum_{n=1}^{\infty}\left(\frac{n}{z}\right) d_{\overline{\chi_{1}}, \overline{\chi_{2}}}(n) K_{0}\left(\frac{2 \pi n}{z \sqrt{p q}}\right) .
$$

Proof. By a simple change of variables, we see that we can rewrite (2.8) in the alternative form

$$
\frac{1}{2 \pi i} \int_{c-i \infty}^{c+i \infty} \Gamma^{2}\left(s+\frac{1}{2}\right) x^{-s} d s=2 \sqrt{x} K_{0}(2 \sqrt{x}), \quad c>-\frac{1}{2},
$$

which we will use twice in the sequel.

Our proof is similar to the two previous proofs. Proceeding as before, but now with the use of (3.11), for $c>\frac{1}{2}$, consider

$$
\begin{aligned}
I: & =\frac{1}{2 \pi i} \int_{c-i \infty}^{c+i \infty}\left(\frac{\pi^{2}}{p q}\right)^{-\left(s+\frac{1}{2}\right)} \Gamma^{2}\left(s+\frac{1}{2}\right) L\left(2 s, \chi_{1}\right) L\left(2 s, \chi_{2}\right) z^{-2 s} d s \\
& =\left(\frac{\pi^{2}}{p q}\right)^{-\frac{1}{2}} \sum_{n=1}^{\infty} d_{\chi_{1}, \chi_{2}}(n) \frac{1}{2 \pi i} \int_{c-i \infty}^{c+i \infty} \Gamma^{2}\left(s+\frac{1}{2}\right)\left(\frac{\pi^{2} n^{2} z^{2}}{p q}\right)^{-s} d s \\
& =\left(\frac{\pi^{2}}{p q}\right)^{-\frac{1}{2}} \sum_{n=1}^{\infty} d_{\chi_{1}, \chi_{2}}(n) 2 \sqrt{\frac{\pi^{2} n^{2} z^{2}}{p q}} K_{0}\left(\frac{2 \pi n z}{\sqrt{p q}}\right) \\
& =2 z \sum_{n=1}^{\infty} n d_{\chi_{1}, \chi_{2}}(n) K_{0}\left(\frac{2 \pi n z}{\sqrt{p q}}\right)
\end{aligned}
$$

We proceed as in the previous two proofs and shift the line of integration to $\sigma=-b, 0<b<\frac{1}{2}$, by integrating over a rectangle with vertices $c \pm i T$ and $-b \pm i T, T>0$, and letting $T \rightarrow \infty$. The argument is the same as that in the proof of Theorem 3.1, but now the restriction $b<\frac{1}{2}$ ensures that the integrand is analytic on the interior of the rectangle. (If we had assumed that $\frac{1}{2}<b<1$, then $\Gamma^{2}\left(s+\frac{1}{2}\right)$ would have a double pole at $s=-\frac{1}{2}$ on the interior of our rectangle.) Hence, shifting the line of integration, using the functional equation (2.5), making 
the change of variable $w=\frac{1}{2}-s$, inverting the order of summation and integration, and appealing to (3.11), we find that

$$
\begin{aligned}
I & =-\frac{1}{2 \pi i} \int_{-b-i \infty}^{-b+i \infty} \frac{\tau\left(\chi_{1}\right) \tau\left(\chi_{2}\right)}{\sqrt{p q}}\left(\frac{\pi^{2}}{p q}\right)^{-(1-s)} \Gamma^{2}(1-s) L\left(1-2 s, \overline{\chi_{1}}\right) L\left(1-2 s, \overline{\chi_{2}}\right) z^{-2 s} d s \\
& =-\frac{\tau\left(\chi_{1}\right) \tau\left(\chi_{2}\right)}{\sqrt{p q}} \frac{1}{2 \pi i} \int_{\frac{1}{2}+b-i \infty}^{\frac{1}{2}+b+i \infty}\left(\frac{\pi^{2}}{p q}\right)^{-\left(\frac{1}{2}+w\right)} \Gamma^{2}\left(\frac{1}{2}+w\right) L\left(2 w, \overline{\chi_{1}}\right) L\left(2 w, \overline{\chi_{2}}\right) z^{-1+2 w} d w \\
& =-\frac{\tau\left(\chi_{1}\right) \tau\left(\chi_{2}\right)}{z \pi} \sum_{n=1}^{\infty} d_{\overline{\chi_{1}}, \overline{\chi_{2}}}(n) \frac{1}{2 \pi i} \int_{\frac{1}{2}+b-i \infty}^{\frac{1}{2}+b+i \infty} \Gamma^{2}\left(\frac{1}{2}+w\right)\left(\frac{\pi^{2} n^{2}}{z^{2} p q}\right)^{-w} d w \\
& =-\frac{\tau\left(\chi_{1}\right) \tau\left(\chi_{2}\right)}{z \pi} \sum_{n=1}^{\infty} d_{\overline{\chi_{1}}, \overline{\chi_{2}}}(n) 2 \sqrt{\frac{\pi^{2} n^{2}}{z^{2} p q}} K_{0}\left(\frac{2 \pi n}{z \sqrt{p q}}\right) \\
& =-2 \frac{\tau\left(\chi_{1}\right) \tau\left(\chi_{2}\right)}{z^{2} \sqrt{p q}} \sum_{n=1}^{\infty} n d_{\overline{\chi_{1}}, \overline{\chi_{2}}}(n) K_{0}\left(\frac{2 \pi n}{z \sqrt{p q}}\right) .
\end{aligned}
$$

Putting (3.13) into (3.12) and manipulating slightly, we complete the proof of (3.10).

We complete our paper with some remarks on $L(1, \chi)$ in connection with the statement of Theorem 3.1 above. Suppose that $\chi$ satisfies the conditions of Theorem 3.1, and in addition suppose that $\chi$ is also a real character. Let us remark that the nonvanishing of $L(1, \chi)$, which plays a well-known role in Dirichlet's theorem on primes in arithmetic progressions, follows from this identity as well and the integral representation (2.9). Indeed, let $z=x$ (real) decrease to zero. From (2.9), we observe that $K_{0}(x)$ is positive and monotonically decreasing on the interval $(0, \infty)$. From the definition (1.1), it is obvious that $K_{0}(x)$ also tends to $\infty$ as $x$ decreases to 0 . At the same time, $d_{\chi}(n)$ is nonnegative for each $n$, which can be seen, for instance, by examining the Euler product on the left-hand side of (1.7). More precisely, the local factors in this Euler product are of the forms $\left(1-p^{-2 s}\right)^{-1}$, $\left(1-p^{-2 s}\right)^{-2}$, or $\left(1-p^{-4 s}\right)^{-1}$, according as to whether $\chi(p)$ equals 0,1 or -1 , respectively. Thus, rewriting the Euler product as a Dirichlet series, we see that all coefficients are nonnegative. It also follows from (1.5) that $d_{\chi}(n) \geq 1$ for each $n$ that is a perfect square. So, as stated above, if $x$ decreases to zero, then the left-hand side of (3.1) tends to $+\infty$. On the other hand, as $x$ tends to infinity, it is clear from (2.10) that $K_{0}(x)$ decays exponentially fast. So the first summand in the series on the right-hand side tends to zero as $x$ decreases to zero. We conclude that the term $\frac{\sqrt{q} L(1, \chi)}{4 x}$ tends to $+\infty$ as $x$ decreases to zero, which implies that $L(1, \chi)$ is strictly positive.

\section{References}

[1] G. E. Andrews and B. C. Berndt, Ramanujan's Lost Notebook. Part IV, Springer, New York, 2013. MR3113512

[2] B. C. Berndt, A. Dixit, and J. Sohn, Character analogues of theorems of Ramanujan, Koshliakov and Guinand, Adv. in Appl. Math. 46 (2011), no. 1-4, 54-70, DOI 10.1016/j.aam.2009.12.003. MR2794013 (2012c:33017)

[3] B. C. Berndt, Y. Lee, and J. Sohn, Koshliakov's formula and Guinand's formula in Ramanujan's lost notebook, Surveys in number theory, Dev. Math., vol. 17, Springer, New York, 2008, pp. 21-42. MR2462944 (2010a:33011)

[4] E. T. Copson, Theory of Functions of a Complex Variable, Oxford University Press, Oxford, 1935. 
[5] H. Davenport, Multiplicative Number Theory, 3rd ed., Graduate Texts in Mathematics, vol. 74, Springer-Verlag, New York, 2000. Revised and with a preface by Hugh L. Montgomery. MR1790423 (2001f:11001)

[6] C. Deninger, On the analogue of the formula of Chowla and Selberg for real quadratic fields, J. Reine Angew. Math. 351 (1984), 171-191, DOI 10.1515/crll.1984.351.171. MR749681 (86f:11085)

[7] A. Erdélyi, ed., Tables of Integral Transforms, Vol. 1, McGraw-Hill, New York, 1954.

[8] W. L. Ferrar, Some solutions of the equation $F(t)=F(t-1)$, J. London Math. Soc. S1-11 (1936), no. 2, 99-103, DOI 10.1112/jlms/s1-11.2.99. MR.1574771

[9] N. S. Koshliakov, On Voronoï's sum-formula, Mess. Math. 58 (1929), 30-32.

[10] S. Ramanujan, The Lost Notebook and Other Unpublished Papers, Springer-Verlag, Berlin; Narosa Publishing House, New Delhi, 1988. With an introduction by George E. Andrews. MR947735 (89j:01078)

[11] E. C. Titchmarsh, The Theory of the Riemann Zeta-Function, Oxford, at the Clarendon Press, 1951. MR0046485(13,741c)

[12] G. N. Watson, Theory of Bessel Functions, 2nd ed., University Press, Cambridge, 1966.

[13] S. Yakubovich, A general class of Voronö̈'s and Koshliakov-Ramanujan's summation formulas involving $d_{k}(n)$, Integral Transforms Spec. Funct. 22 (2011), no. 11, 801-821, DOI 10.1080/10652469.2010.540447. MR 2842562

Department of Mathematics, University of Illinois, 1409 West Green Street, UrBANA, ILLINOIS 61801

E-mail address: berndt@illinois.edu

Department of Mathematics, Ohio State University, Columbus, Ohio 43210

E-mail address: kim.1674@math.osu.edu

Department of Mathematics, University of Illinois, 1409 West Green Street, Urbana, Illinois 61801 - And - Institute of Mathematics of the Romanian Academy, P.O. Box 1-764, Bucharest RO-70700, Romania

E-mail address: zaharesc@illinois.edu 